\title{
Using MILOS to build a Multimedia Digital Library Application: The PhotoBook experience *
}

\author{
Giuseppe Amato, Paolo Bolettieri, Franca Debole, Fabrizio Falchi, Fausto Rabitti, and \\ Pasquale Savino \\ ISTI-CNR, Pisa, Italy \\ firstname.lastnamedisti.cnr.it
}

\begin{abstract}
The digital library field is recently broadening its scope of applicability and it is also continuously adapting to the frequent changes occurring in the internet society. Accordingly, digital libraries are slightly moving from a controlled environment accessible only to professionals and domain-experts, to environments accessible to casual users that want to exploit the potentialities offered by the digital library technology. These new trends require, for instance, new search paradigms to be offered, new media content to be managed, and new description extraction techniques to be used.

Building digital library applications, and effectively adapting them to new emerging trends, requires to develop a platform that offers standard and powerful building blocks to support application developers. In this paper we discuss our experience of using MILOS, a multimedia content management system oriented to the construction of digital libraries, to build a demanding application dedicated to non-professional users. Specifically, we discuss the design and implementation of an on-line photo album (PhotoBook), which is a digital library application that allows people to manage their own photos, to share them with friends, and to make them publicly available and searchable.

PhotoBook, uses a complex internal metadata schema (MPEG-7) and allows users to simply express complex queries (combining similarity search and fielded search), enabling them to retrieve material of interest even if metadata are imprecise or missing.
\end{abstract}

\section{Introduction}

Many Digital Library Applications (DLA) supporting an effective and efficient archiving and retrieval of documents with different types of data and content, and that are used for many diverse purposes, have been developed. However, Digital Library technology will arrive at a definitive maturity when powerful and simple software development tools that enable the development of DLAs, will be available. Regrettably, nowadays several Digital Library Applications are monolithic software modules where the application itself, the content management software, and the digital library are merged

\footnotetext{
* This work was partially supported by the DELOS NoE, funded by the European Commission under FP6 (Sixth Framework Programme), by the ECD and VICE projects, both funded by the Italian Ministry of Research and Education. The PhotoBook Application can be accessed from http://milos.isti.cnr.it/
} 
together. Many systems were built having in mind a specific application and, in many cases, a specific document collection, thus resulting in an ad-hoc solution: all components of the DLA - the data repository, the metadata manager, the search and retrieval components, etc. - are specific to a given application and cannot be easily used in other environments. In these cases the development of the DLA is expensive and it is not possible to personalize or specialize the DL services and to adapt them to new user requirements.

An important building block of any DLA is the module responsible for the archiving, access, and retrieval of data objects. In many cases, as types of data managed change, or when different metadata format are used, or if different search functionality is required, a new component is build. Standard building components to help digital library application developers are needed.

The aim of this work is to illustrate the use of MILOS, a Multimedia Content Management System (MCMS) for digital libraries, to develop a demanding application: the PhotoBook Digital Library (http://milos.isti.cnr.it).

The advent of digital photography, combined to the wide access to internet resources, made popular the creation of personal and publicly accessible distributed photo archives. Typical examples are the Flickr service (www.flickr.com), the snapfish service offered by HP (www.snapfish.com), and Picasa (picasa.google.com). Users of these archives may create and manage their own photo albums, decide who and how can access their photos, provide a description of the photos to simplify their access. This simple yet powerful application, poses several complex requirements to the MCMS component, which must support: (i) the distributed storage and classification of photos, (ii) the description of photo content through an appropriate metadata model, (iii) the search based on photo description and photo content, (iv) the management of personal folders (photo albums), (v) the controlled access management. Some of the mentioned functionality, do require the development of an appropriate Web-based user interface. A final, but significant requirement is that the development of the application must be simple and fast. The tools previously mentioned (flickr, snapfish, and Picasa) offer most of these functionality but with search capabilities limited to manually associated metadata tags. Furthermore, as mentioned at the beginning of this paper, they are ad-hoc applications, whose development required significant investments and whose extensions would also require considerable efforts. Our aim is to show how, by using the general purpose MCMS system MILOS, is possible to build an application with similar functionality and even with more powerful search capabilities - with a limited effort.

The development of the Photobook application has a twofold purpose: i) testing how the most recent digital library technology is able to deal with these new emerging trends, and ii) to verify if the MILOS multimedia content management system is capable of providing effective tools to rapidly develop a multimedia digital library application with high demanding requirements.

In the following we will briefly resume the functionality of the MILOS MCMS. We will discuss the functionality of the PhotoBook Digital Library Application. Then, we will present the architecture of PhotoBook and the specific functionality offered and the solutions adopted for picture management, metadata management - with particular attention to automatic metadata extraction - and photo search. 


\section{MILOS Overview}

The MILOS system $[4,1]$ is a Multimedia Content Management System with a number of characteristics that make it particularly suitable for the development of Digital Library applications. In particular, as we will underline in this section, MILOS functionality are particularly appealing for the development of the Photobook application, which requires the archiving of a large number of images from users distributed over the internet, efficient content-based retrieval of photos, according to their metadata values and based on their physical content, creation of a Web interface for the storage, query formulation and presentation of results.

Key characteristics of the MILOS system are the flexibility in managing different types of data and metadata and the independence from the specific format used to represent them. This implies that the application developer is not required to specify the details of the storage strategies used and the details of the access methods to be adopted; he/she only needs to specify the characteristics of the data and metadata and the functionality that are required, such as the requirement of storing high resolution photos, and supporting their efficient access based on the combination of metadata attributes and physical characteristics of photos. The flexibility of the MILOS system is also related to the possibility of developing end-user applications which are independent from the modality used to store data and search them. In particular, it is possible to store data described with a specific metadata model and to search them by using a different model.

Another key characteristic of MILOS is the efficiency in storing and searching multimedia objects. This requires a system which is (a) scalable when the size of the archive and the number of users accessing the application varies, and (b) efficient when processing complex queries on metadata values and data object's content.

The MILOS system has three main components: the Metadata Storage and Retrieval (MSR) component - dedicated to support the storage of metadata and the content-based retrieval of multimedia objects - the Multi Media Server (MMS) component - dedicated to support the storage and access of multimedia content - and the Repository Metadata Integrator (RMI) component - dedicated to provide the independence between the metadata format used by end-user applications and the internal metadata formats.

Let us provide some more details on the MSR, which is of particular importance in the PhotBook application. Details on the MMS and RMI are provided elsewhere [4].

\subsection{Metadata Storage and Retrieval}

The MILOS MSR, despite the approaches adopted by other similar systems, uses an enhanced native XML database/repository system with special features for DL applications. Indeed, XML represented metadata may have arbitrary complex structures, which allows one to deal with complex metadata schemas, and can be easily exported and imported.

The MILOS XML database [2] stores and retrieves any valid XML document. No metadata schema or XML schema definition is needed before inserting an XML document, except optional index definition for performance improvement. Once an arbitrary XML document has been inserted in the database it can be immediately retrieved. This allows DL applications to use arbitrary (XML encoded) metadata schemas and to deal 
with heterogeneous metadata, without schema design constraints and/or overhead due to metadata translation.

This special purpose native XML database/repository system is much simpler than a general purpose commercial XML database system, but with much better performances where needed. It supports standard XML query languages (XPath [6] and XQuery [7]) and it offers advanced search and indexing functionality on XML documents. The MILOS XML database supports high performance search and retrieval on heavily structured XML documents, relying on specific index structures [3,14], as well as full text search [13], automatic classification [8], and feature similarity search [15,5]. This is particularly relevant in the Photobook application, where metadata photos are represented in the MPEG-7 format: each photo includes in the description also features automatically extracted from visual properties, such as color histograms, textures, shapes, etc.

To deal easily and transparently with these advanced search and indexing functionalities, the syntax of the basic XQuery language was extended to deal with approximate match and ranking. Specifically the $\sim$ operator is used for approximate match (that is, feature similarity and text search). More details can be found in [2].

\section{The PhotoBook digital library application}

The use of digital cameras is becoming very popular in the society. People can take picture and immediately see the result by downloading the images on their personal computers, avoiding the cost of printing them. Digital technology applied to the personal cameras has changed the way people use and manage their pictures. People take much more pictures, given that they can immediately see them at no cost, print a significant minority of them, and especially share them with friends by sending them by email, or more frequently by storing and publishing them on on-line photo albums.

Several sites that offer popular on-line photo albums services are nowadays available on the internet and they are continuously enriched by new services to help people on one hand to publish and share their material, on the other to access and search for published material.

Even if on-line photo albums are mainly addressed to non-professional users, they can be reasonably considered as digital library applications. In fact, such systems typically offer important functionality also offered by professional digital library systems. As an example, on-line photo albums offer storage and preservation services, privacy and rights management, personalization, metadata editing and annotation functionality, advanced search functionality.

Two distinct types of users can be broadly identified for such systems. Users can act as publishers when they publish, annotate, classify, and manage digital photos. Users can act as searchers when they search for published digital material. Both publishers, and searchers are non-professional users of these on-line services. The two roles sometime can be merged. Many people are in fact at the same time publishers and searchers.

On-line photo albums nowadays represent an emergent phenomenon of the internet society. Several stand-alone on-line photo album services are in fact available on the 
internet. In addition, almost all internet providers, portals, search engines, photo camera producer, and photo printing services have added an on-line photo albums to the services that they already provide.

In the following we discuss the requirements of the PhotoBook (http://milos.isti.cnr.it/) application, its architecture and the various components.

\subsection{PhotoBook Application Functionality}

The PhotoBook application is intended to be accessible on-line by everybody. It offers services to two categories of users: non-registered users and registered users.

Non-registered users can use the PhotoBook application to search for and access photos that were uploaded in the system and made publicly available.

Registered users in addition to the capabilities of the non-registered users can also

1. upload photos in the system

2. manage their own photos

3. share their photos with friends

4. make their photo publicly available

Each photo managed by the PhotoBook has one single owner, which is the user that uploaded it (we suppose that a person that uploads a picture owns it or he/she has the right to do it). The owner can set a photo to be private or public.

All users (registered and non-registered) should be allowed to search and access digital pictures that were uploaded in the system and that were marked by the owner as public.

Registered users can organize their own photos in albums. An album is basically a collection of photos. For instance, a user can decide to create an album containing the photos that he/she took during last summer. A photo may belong to several albums at the same time. Users can share an album (containing both private and public photos) with friends. A private photo can be accessed just by the owner of the photo and by his friends, when they are given an handler to an album that contains it. A user's friend does not need to be registered in the system. Access to an album is obtained by using a system-generated URL containing the handler to the album. Every non-registered user can access an album using the album handler.

The owner of an album can remove an album at any time. An album deletion does not delete the photos that it contains, which must be explicitly removed from the system if needed.

When a registered user uploads a new photo, he/she can associate it with some descriptions related to the picture's content. Descriptions can be changed and refined afterward. A bulk upload functionality is also supported to easily insert photos having common descriptions. For instance, a user can insert, with a single action, all photos taken during last weekend in the mountains. In this case, all inserted photos are associated with the same common description.

In addition to descriptions created by the users, the system automatically analyzes photos to extract additional metadata. Specifically, feature descriptors that enable similarity search are automatically extracted. Similarity search [15] allows users to search 
for pictures similar to pictures chosen as queries. This possibility can be particularly useful to retrieve poorly described pictures. Consider that, as previously stated, the PhotoBook application is addressed to a non professional target. Therefore, imprecise, erroneous, incomplete, or completely missing descriptions may frequently occur. Similarity search is an option for searching for photos of interest, which is really useful especially in this non-professional context. With similarity search, a user can be able to retrieve, for instance, pictures of the tour Eiffel by using another picture of the tour Eiffel as a query, even if the retrieved pictures were not correctly annotated by their owner.

Metadata manually and automatically generated are represented by using standard metadata schema. Specifically we have used MPEG-7 [9] metadata schema, given that it is able to represent both low level feature descriptors, for similarity search, and more conceptual descriptions. However, given that the PhotoBook application is intended to be used by non professional users, the metadata complexity is hidden to them. This implies that information is presented to the user in a natural and intuitive way and that users are asked to insert a minimal amount of descriptions when uploading photos. A relevant part of information is obtained automatically, and the system is able to satisfy most of the user requirements even with incomplete, erroneous, or partial information. For instance, in addition to image analysis to support similarity search, a lot of more conceptual information can be obtained exploiting file names and folders names.

During photo management and photo searching activities, users see on the screen the photo thumbnails. However, a user can download full size pictures, if needed.

\subsection{PhotoBook Architecture}

The architecture of the PhotoBook application is sketched in Figure 1. It is a classical $\mathrm{n}$-tier application, where the data layer, the application layer, the presentation layer, and the client layer are responsible of different classes of activity of the application.

At the data layer we have MILOS, which is responsible for the management, access, and retrieval, of all types of data of the application. Data managed by MILOS include pictures uploaded by users, metadata, and user data.

At the application layer we have a set of components responsible of mediating the interaction of the modules that implement the web user interface with MILOS. These components actually implement the application's logic, separately and independently from the user interface logic and the data management logic. These components are responsible of checking the credentials of users, organizing retrieved result on behalf of the application logic, translating the user queries into correct queries to MILOS, building valid metadata on the basis of the user input, implementing storage strategies for the uploaded material (relying on the MILOS features), and generating additional metadata by analyzing uploaded data. Interaction with MILOS is obtained using the SOAP protocol, being MILOS a Web service. These components are also implemented as web services.

The presentation layer contains the software modules in charge of drawing the user interface on behalf of the client layer. PhotoBook is a web application: accordingly, the interface layer is composed of Java Server Page (JSP) modules which dynamically generate the web user interface. These modules interact with the application layer by using SOAP. 


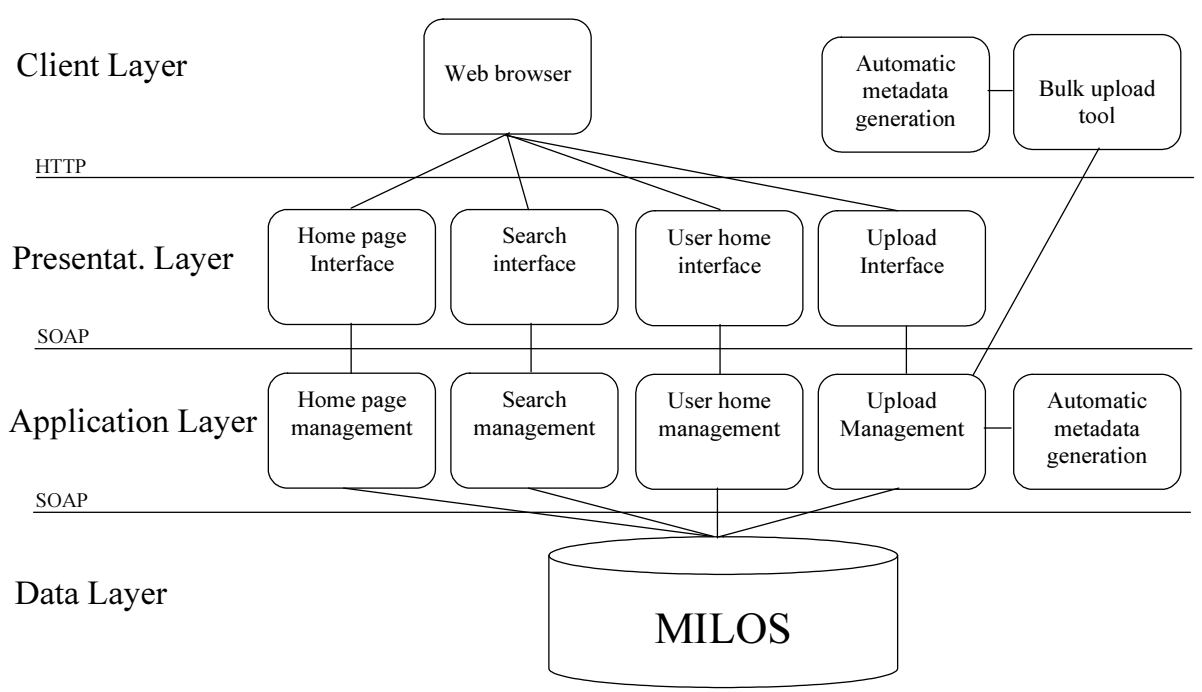

Fig. 1. Architecture of the PhotoBook Application

Clients access PhotoBook by using a normal web browser. In addition at the client side, users may optionally use a Java bulk-load component that allows them to upload several pictures in the system, and to locally analyze them to automatically generate additional metadata. The use of the bulk-load tool, moves part of the logic for metadata generation to the client side, distributing the burden of image analysis among several clients and improving upload performance of the system.

\subsection{The PhotoBook user interface}

A sketch of the main web interfaces is shown in Figure 2. The home page of the PhotoBook application is shown in Figure 2a. From the home page, every user (registered and non-registered) can search for public material on the system, login for managing the owned material, registering into the system. From the home page users can search for pictures by using a fielded search or similarity search. In case of fielded search users can search for pictures by expressing restrictions on the owner of the pictures, the location where they were taken, their title, and on the textual description of the pictures. In case of similarity search, the user can search by choosing a picture among those randomly proposed by the system. Random pictures can be renewed on demand by the user.

Results are shown in the search page (Figure 2b). From there, users can refine their queries by choosing a picture in the result to submit a new similarity search or to submit a complex search query, which combines similarity and fielded search. For instance, a user can search for images similar to the chosen one, whose location is Pisa.

Registered users that enter the system (Figure 2c), can also manage their own collection of pictures. Registered users can update metadata associated with pictures, organize pictures in thematic albums, share albums with friends, and upload new pictures. 


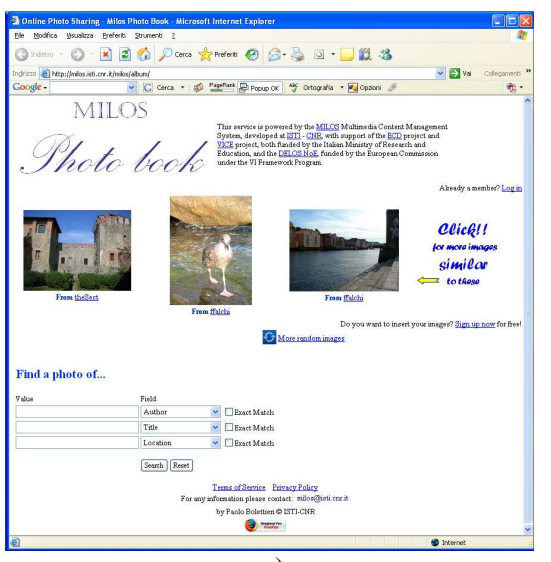

a)

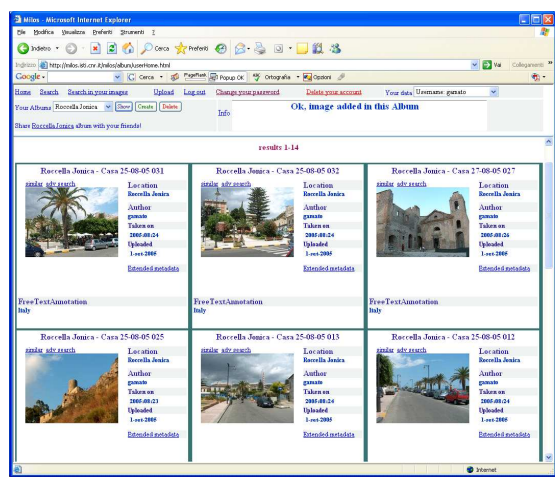

c)

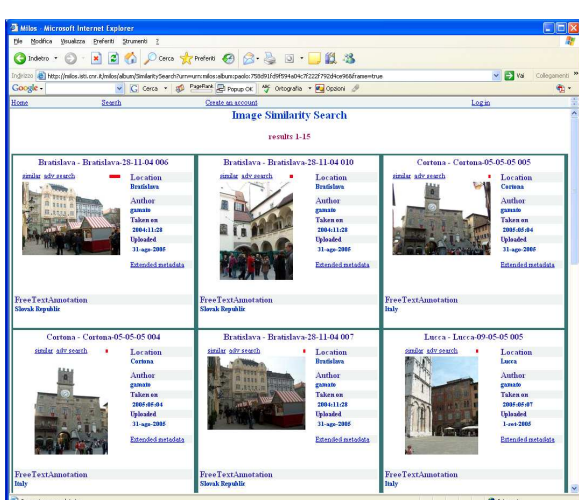

b)

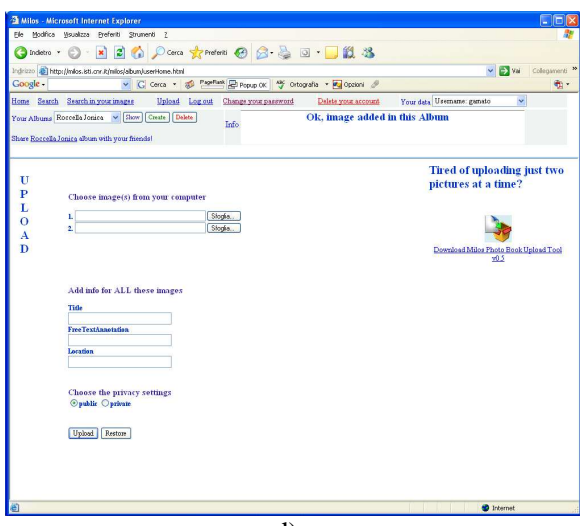

d)

Fig. 2. The PhotoBook interface (http://milos.isti.cnr.it). a) The home page of the PhotoBook application, b) The search interface of the PhotoBook application, c) The registered user page of the PhotoBook application, d) The upload page of the PhotoBook application

There are two options for uploading new pictures. Registered users can use the web interface (Figure 2d) or a bulk-load tool running on their computers. By using the web interface users can upload up to two pictures at time, by specifying common descriptions for both. In this case, automatic metadata extraction for similarity search is executed on the PhotoBook server. By using the bulk-load tool, users can submit an arbitrary amount of pictures from their hard disk. In this case, users can assign common descriptions to the uploaded picture. In addition the bulk-load tool can refine these description by using information extracted from the folder names and file names. Image analysis for similarity search is also performed on the user computer. By using the upload tool, the PhotoBook server has just to register the insertions, store the pictures, and update the internal indexes. 


\section{Picture management}

MILOS allows different storage strategies to be used when dealing with different types of media. The use of specific storage strategies is transparent to the application. To obtain that, MILOS (the MMS component) identifies all documents with an URN and maintains a mapping table to associate URN with actual storage locations and access protocols. When an application requires to retrieve a document, it uses the URN to make the request to the MMS, which returns the URL to be used to access the document.

In the PhotoBook application we decided to store pictures in a normal file system, and to access them via a web server using the HTTP protocol. Pictures belonging to a user are stored in a personal folder. Two different versions of every pictures are stored: a thumbnail version and a full size version. Thumbnails and full version pictures are stored in two different subfolder of the user's folder.

An example of URN used in the PhotoBook is the following:

urn:milos:album:Paolo:image_jpeg:7897056ecc55cd6f7cffa78413e4e2ac

which refers to a jpeg image of the user Paolo. When the PhotoBook application wants to retrieve a picture, it uses the getURL(URN,version) method of MILOS to obtain the URL to be used to retrieve it. When a request for the full size version of previous URN is receioved, MILOS returns the following URL:

http://milos.isti.cnr.it/milos-MMS/MMS/album/ Paolo/big/image_jpeg/

/image_jpeg7897056ecc55cd6f7cffa78413e4e2ac.jpg

\section{Metadata Management}

MILOS is able to deal with any metadata schema. The only requirement is that metadata should be encoded in XML. No schema definition is needed to instruct MILOS to deal with a specific metadata schema. Once an XML document is inserted in MILOS, it is immediately available to be searched and accessed, using the advanced search functionality of the native XML search engine embedded in MILOS.

This feature gave us a lot of freedom and flexibility in the choice of the metadata to be used for the application and freed us from the difficulties of instructing a specific search engine or database to deal with them.

We decided to use MPEG-7 metadata [9] for the image description. Figure 3a gives an example of the MPEG-7 description of a picture. An MPEG-7 description contains low level features to be used for similarity search, conceptual content descriptions, usage rights, creation time information, etc. Specifically, the <VisualDescriptor $>$ tags, in the figure, contain scalable color, color layout, color structure, edge histogram, homogeneous texture information to be used for image similarity search. MILOS indexes this tag with a special index to offer efficient similarity search. Conceptual information is contained in the $\langle$ Title $\rangle$, $\langle$ Abstract $\rangle$, and $\langle$ Location $>$ tags. Right management information is contained in tags $\langle$ Usage Information $\rangle$. Creation time information is maintained in $\langle$ CreationTool $>$ tag. Specifically, eXtended Image File Format (XIFF) information is automatically extracted from pictures. This information includes, for instance, parameters set in the camera when the photo was taken, the 


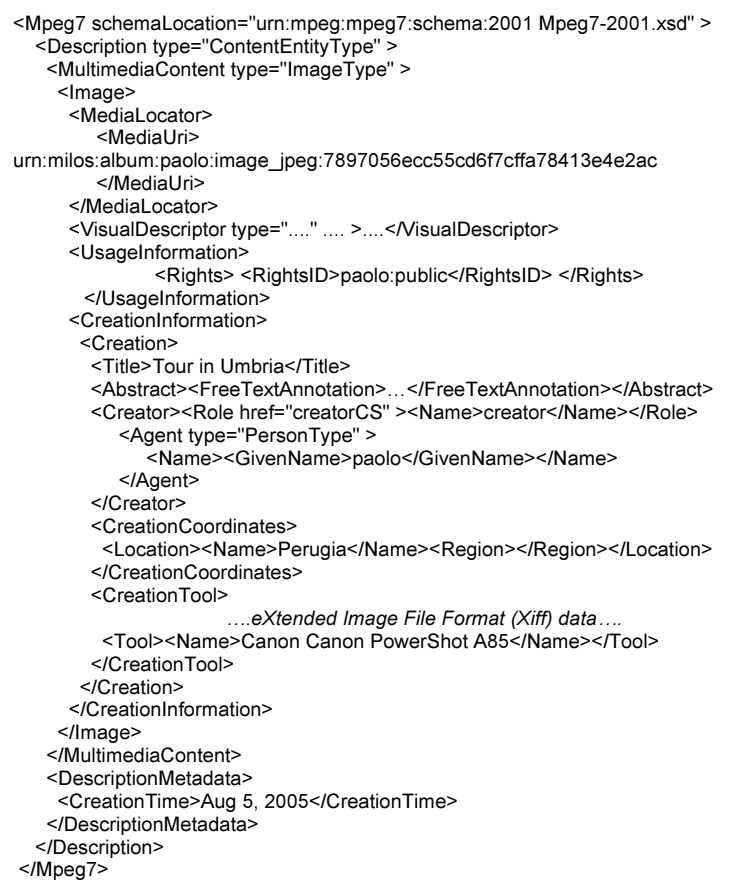

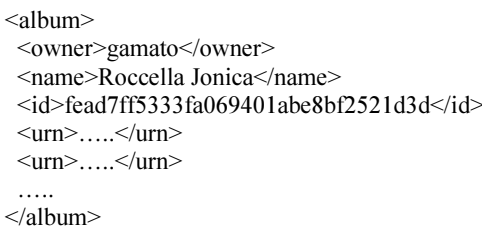

c)

Fig. 3. Metadata used in the PhotoBook application: a) Mpeg7 metadata to describe the pictures, b) representation of albums (thematic collection pictures), c) user data

type of camera, etc. The MPEG-7 standard prescribes that metadata must be encoded in XML. Therefore, its use was straightforward in MILOS.

Users can create thematic albums containing pictures related for instance to an event. Albums are also encoded in XML as shown in Figure 3b. An album has basically a name, an owner, and a set of pictures included in the album.

User data are also very simple. Figure $3 \mathrm{c}$ shows how they are represented in PhotoBook. A user has a username, a password, and an email address.

\subsection{Automatic image processing}

Feature extraction was performed employing an application we built upon the MPEG-7 experimentation model (XM, [11]) of MPEG-7 Part 6: Reference Software. The software can extract all MPEG-7 image Visual Descriptors defined in [10]. For the PhotoBook we extract 5 MPEG-7 descriptors: ScalableColor (a color Histogram in the HSV Color Space), ColorStructure (captures both color content and information about the spatial arrangement of the colors), ColorLayout (represents the spatial layout of color images), EdgeHistogram (spatial distribution of five types of edges), and HomogeneousTexture (characterizes the properties of texture in an image). For all thoose descriptors the suggested distance functions [12] are metric. The result of the extrac- 
tion process is an XML document like the one in Figure 3 without usage and creation information. The values inside the $\langle\mathrm{Vi}$ isualDescriptor $>$ tags are integer vectors (ScalavbleColor, ColorSTructure and EdgeHistogram) or more complicated XML subtrees with integers as values (ColorLayout and HomogeneousTexture).

\section{Search capabilities}

The MILOS native XML database/repository supports high performance search and retrieval on heavily structured XML documents, relying on specific index structures [3, 14], as well as full text search [13], automatic classification [8], and feature similarity search [5]. This is compatible with current trends of the new generation of XML encoded metadata standards, such as MPEG-7, which include in their description also features automatically extracted from visual documents, such as color histograms, textures, shapes, etc. Specifically, the MILOS XML database allows the system administrator to associate specific XML element names with special indexes. Therefore, for instance, the tag name <abstract $>$ can be associated with a full text index. On the other hand, the MPEG-7 <VisualDescriptor $>$ tag can be associated with a similarity search index structure.

These features of the MILOS system were very useful to provide users of PhotoBook with advanced search functionalities and to provide developers with all needed functionality for picture description, album, and user management.

Specifically the PhotoBook application allows users to use similarity search, by exploiting the $<$ VisualDescriptor $>$ tags included in the MPEG-7 metadata. Users can also submit full-text queries, by using the full-text descriptions included in the <abstract $>$ tags. In addition, users can perform a search by expressing queries that use any tag content of the MPEG-7 metadata.

Users can also express complex queries, where full-text, fielded, and similarity search is conveniently combined.

\section{Conclusions}

This paper illustrates the main characteristics, architecture and design choices adopted in the PhotoBook Digital Library application, which supports archiving, indexing, sharing and content-based search of photos. PhotoBook was built by using a general purpose Multimedia Content Management System, MILOS, which is specifically designed to create high performance Digital Library applications. By using MILOS we had several advantages: (a) the development of the entire application was realized with a limited effort - approximately one month of work of an experienced programmer, that developed the user interface of the application, (b) powerful and efficient content-based search capabilities have been included, (c) flexible storage management is possible - for example, if the size of the archive will increase in the future, the storage strategies can change without any modification to the application and transparently for end-users, (d) integration with other similar archives, based on different metadata formats, or archiving of photos represented in metadata formats different from that used in PhotoBook, can be easily obtained. 
Future research efforts will be spent in extending some MILOS functionality: the development of the PhotoBook application has shown that tools to be used for the development of the application user interface would greatly reduce application development costs; on the other side, by improving the automatic semantic analysis of multimedia content, it should be possible to support more powerful content-based searches.

The extended MILOS functionality will then be used to provide a new version of the PhotoBook application, with the extended classification, indexing and search capabilities. Finally, it is our intention to continue the test of the MILOS system by developing other Digital Library applications.

\section{References}

1. MILOS: Multimedia dIgital Library for On-line Search. http://milos.isti.cnr.it/.

2. G. Amato and F. Debole. A native xml database supporting approximate match search. In Europeean Conference on Digital Libraries, ECDL 2005, Vienna, AT, September 18-23 2005, 2005.

3. G. Amato, F. Debole, F. Rabitti, and P. Zezula. YAPI: Yet another path index for XML searching. In ECDL 2003, 7th European Conference on Research and Advanced Technology for Digital Libraries, Trondheim, Norway, August 17-22, 2003, 2003.

4. G. Amato, C. Gennaro, F. Rabitti, and P. Savino. Milos: A multimedia content management system for digital library applications. In Europeean Conference on Digital Libraries, ECDL 2004, Bath, UK, September 12-17 2004, 2004.

5. C. Böhm, S. Berchtold, and D. Keim. Searching in high-dimensional spaces: Index structures for improving the performance of multimedia databases. ACM Computing Surveys, 33(3):322-373, September 2001.

6. W. W. W. Consortium. XML path language (XPath), version 1.0, W3C. Recommendation, November 1999.

7. W. W. W. Consortium. XQuery 1.0: An XML query language. W3C Working Draft, November 2002. http://www.w3.org/TR/xquery.

8. N. Cristianini and J. Shawe-Taylor. An Introduction to Support Vector Machines. Cambidge University Press, 2000.

9. ISO/IEC. Information technology - Multimedia content description interfaces. 15938.

10. ISO/IEC. Information technology - Multimedia content description interfaces. Part 3: Visual. 15938-3:2002.

11. ISO/IEC. Information technology - Multimedia content description interfaces. Part 6: Reference Software. 15938-6:2003.

12. P. Salembier and T. Sikora. Introduction to MPEG-7: Multimedia Content Description Interface. John Wiley \& Sons, Inc., New York, NY, USA, 2002.

13. G. Salton and M. J. McGill. Introduction to Modern Information Retrieval. McGraw-Hill Book Company, 1983.

14. P. Zezula, G. Amato, F. Debole, and F. Rabitti. Tree signatures for xml querying and navigation. In Database and XML Technologies, First International XML Database Symposium, XSym 2003, volume 2824 of LNCS, pages 149-163. Springer, 2003.

15. P. Zezula, G. Amato, V. Dohnal, and M. Batko. Similarity Search - The Metric Space Approach, volume 32 of Advances in Database Systems. Springer, 2006. 